\title{
Stable and Efficient Portfolios
}

\author{
Apostolos Kourtis*
}

This version: January 15, 2013

\begin{abstract}
DeMiguel et al. (2009a) show that estimation errors lead to mean-variance portfolios that underperform naive diversification $(1 / N)$. In response to this finding, new studies have developed more efficient sample-based portfolio strategies that offer higher risk-adjusted returns than $1 / N$. In this paper, I show that the latter portfolio may still be superior in the presence of transaction costs. This is because sample-based strategies tend to be unstable over time. Instability leads to high transaction costs that significantly reduce portfolio returns. I resolve this issue by introducing a stability penalty to the standard mean-variance objective that controls the deviation from the investor's portfolio before rebalancing. In contrast to other techniques in the literature that account for transaction costs, the stability penalty offers intuitive analytical solutions to the portfolio choice problem. As such, it allows to treat estimation errors and transactions costs in a joint manner and it can be directly applied to any portfolio strategy. I evaluate the stability approach by applying it to nine sample-based strategies from the literature and study their performance in five data sets of monthly returns. The results confirm that it leads to stable and efficient portfolios that offer higher Sharpe ratio than $1 / N$ and equal or lower turnover.
\end{abstract}

Keywords: Portfolio Optimization, Stability, Estimation Risk, Transaction Costs

JEL Classification: C13, C51, C61, G11

\footnotetext{
${ }^{*}$ Norwich Business School, University of East Anglia, Norwich Research Park, Norwich, Norfolk, NR4 7TJ, UK a.kourtis@uea.ac.uk. Tel. +44 (0)1603 591387
} 


\section{Introduction}

The mean-variance theory of Markowitz (1952) is the most important portfolio choice model in academia and investment practice. It provides simple and analytically tractable portfolios of minimum risk for a desired level of expected return. Practical applications though suffer from two critical issues. First, the parameters that define optimal portfolios are unknown and need to be estimated in finite samples. Estimation errors insert risk in the portfolio selection process, coined as estimation risk in the literature, which may significantly reduce out-of-sample performance (e.g., see Michaud, 1989; Best and Grauer, 1991). Second, the portfolio weights tend to change dramatically over time. This instability translates into high transaction costs which further decrease portfolio returns. In this paper, I treat estimation risk and instability in a joint manner.

With regards to estimation risk, a vast literature has developed around providing more efficient estimators of the unknown parameters or the optimal weights (Brandt, 2009, provides an excellent review). This literature has been recently challenged by the work of DeMiguel et al. (2009a) who find that $1 / N$ outperforms mean-variance portfolios and most of their extensions. In response to this finding, DeMiguel et al. (2009b), Tu and Zhou (2011), Kirby and Ostdiek (2012) and Kourtis et al. (2012) develop new sample-based portfolio strategies that offer significantly higher riskadjusted returns than $1 / N$. I show that the latter portfolio may still be superior in the presence of transaction costs. This is because most of the sample-based strategies are significantly more unstable than $1 / N$. Notably, the approaches of DeMiguel et al. (2009b), Tu and Zhou (2011) and Kourtis et al. (2012) lead to negative Sharpe ratios in several cases once I account for proportional transaction costs of 100 basis points (bp). For example, in a dataset of 8 international market portfolios the strategies of Demiguel et al. (2009b) and Kourtis et al. (2012) result in an annual Sharpe ratio of 0.822 and 0.926 , respectively, in the absence of transaction costs. The Sharpe ratio of $1 / N$ is lower (0.542). Once I account for transaction costs, though, the two sample-based strategies respectively lead to Sharpe ratios of -0.076 and -0.373 while the Sharpe ratio of $1 / N$ is 0.522. This is because the turnover of the two sample strategies is up to 70 times higher. Such results highlight the importance of portfolio stability.

The standard approach to promote stability is to directly incorporate transaction costs in the portfolio optimization problem. Numerous studies modify the original mean-variance model to account for fixed and/or variable transaction costs (Woodside-Oriakhi et al., 2013, provide a recent review of this literature). Due to the nature of transaction costs, the analytical derivation of the portfolio weights is no longer feasible and a computational algorithm is required instead. However, such algorithms may be inefficient for a large number of assets. Moreover, the lack of an analytical representation of the optimal weights does not allow to apply such approaches to the recent advances in the literature of estimation risk discussed above. This is important in light of the finding of Kirby and Ostdiek (2012) that estimation risk has a magnifying effect on transaction costs.

In this paper I develop a method to increase the stability of sample-based strategies while maintain their efficiency. I propose to impose a "stability penalty" directly to the investor's objective. The penalty controls the deviation of the portfolio weights after rebalancing from the weights be- 
fore rebalancing. The novelty is that I measure the deviation using the $\Sigma^{\frac{1}{2}}$-norm instead of the 1-norm that is usually applied in the literature, where $\Sigma$ is the covariance matrix of asset returns. This leads to an intuitive analytical solution to the portfolio optimization problem; the "stable" portfolio weights are a linear combination of the weights before and after rebalancing.

My approach effectively treats estimation risk since the form of the weights allows the application to any portfolio strategy from the estimation risk literature. Moreover, I show that the stability penalty may improve performance even in the absence of transaction costs. This is because the stability penalty can be interpreted in two interesting manners. First, it is equivalent to imposing a norm constraint in the original mean-variance problem. DeMiguel et al. (2009b) show that such constraints can increase risk-adjusted returns in the presence of transaction costs. Second, the stability penalty is equivalent to shrinking the portfolio weights to that of a buy-and hold strategy. The latter may be efficient when the market is in momentum (i.e. asset returns are positively autocorrelated).

The main feature of my method is that it can be used to reduce the turnover of any sample-based portfolio strategy. The extent of the turnover reduction is controlled by a stability parameter. I provide two alternatives for its selection. First, I choose the parameter that matches the turnover of the strategy to that of $1 / N$. This is because the latter in known to result in low turnover while being a natural benchmark in this study. Moreover, it is possible to derive the distribution of the turnover of $1 / N$ under standard assumptions. This is useful since it allows the estimation of the expected transaction costs of the stable portfolios or other statistical inference about the portfolio stability. Second, I choose the parameter that maximizes the portfolio return of the previous month. This is motivated by the positive autocorrelation of portfolio returns (see, Campbell et al., 1997, DeMiguel et al., 2009b, Kourtis et al., 2012) and may lead to further performance improvements.

To evaluate the stability approach, I apply it to nine sample-based srategies from the literature. These include three portfolios from the sample-based mean-variance frontier, a short-sale constrained mean-variance portfolio, the shrinkage-based portfolio of Ledoit and Wolf (2004a) as well as five portfolios from the studies of DeMiguel et al. (2009b), Tu and Zhou (2011), Kirby and Ostdiek (2012) and Kourtis et al. (2012). In this manner, I derive stable versions of these sample-based strategies. I next compare their out-of-sample performance to that of $1 / N$ and of their "unstable" counterparts in 5 datasets of real asset returns. I decompose out-of-sample performance into efficiency and stability; the first is measured by Sharpe ratio and the second by the average portfolio turnover. I compute the Sharpe ratio in the case of no transaction costs and for transaction costs of $100 \mathrm{bp}$.

The results of the empirical analysis confirm that the stability penalty leads to both efficient and stable portfolios. Revisiting the dataset of 10 industry portfolios discussed above, I find that the stable versions of the portfolios of DeMiguel et al. (2009b) and Kourtis et al. (2012) offer a Sharpe ratio of 0.600 and 0.629 respectively outperforming $1 / N$ under transaction costs of $100 \mathrm{bp}$. At the same time, all stable strategies generate a turnover equal or lower to $1 / N$ by construction. Moreover, in three out of the five datasets the stability approach improves portfolio performance 
even in the absence of transaction costs. For instance, in a set of 8 international market portfolios the Sharpe ratio of the strategies of DeMiguel et al. (2009b), Tu and Zhou (2011) and Kourtis et al. (2012) increases from $0.288,0.147$ and 0.168 to $0.368,0.324$ and 0.426 when the stability penalty is applied. The latter values are higher than the Sharpe ratio of $1 / N$ at 0.344 . Out of the two methods for selecting the stability parameter, the momentum-based criterion leads in higher Sharpe ratios than fixing the parameter to match the turnover of $1 / N$ in most cases.

The remaining of this work is organised as follows. Section 2 presents the mean-variance framework. The effects of estimation risk and portfolio instability to the performance of several portfolio strategies are discussed in Section 3. Section 4 develops an approach to stabilize portfolio strategies and presents its merits. Section 5 evaluates the stability approach in several datasets of real asset returns while Section 6 concludes this paper.

\section{The mean-variance framework}

I present the mean-variance framework under different portfolio constraints. I consider a multiperiod investor in a market of $N$ risky assets and risk-free asset. The returns on the risky assets over the period $t$ are $R_{t}$ while $R_{t}^{f}$ stands for the risk-free return. At each time $t$, the optimal weights $w_{t}$ for the investor minimize the variance of the portfolio excess returns

$$
\min _{w_{t}} w_{t}^{\prime} \Sigma w_{t}
$$

subject to the constraints

$$
\begin{gathered}
w_{t}^{\prime} \mu=\mu^{0} \\
w_{t}^{\prime} 1_{N}=1
\end{gathered}
$$

In the above, $\mu$ and $\Sigma$ are respectively the ex-ante mean and covariance matrix of the excess returns $r_{t}=R_{t}-r_{t}^{f}$. To simplify notation, I assume that these moments are time-invariant. However, all results can be readily extended to the case of time-varying moments. The first constraint sets the the level of return required by the investor $\left(\mu_{0}\right)$. Allowing $\mu^{0}$ to take different values generates the minimum variance frontier. The second constraint ensures that the investor only holds the risky assets ( $1_{N}$ is an $N$-dimensional vector of 1 's). I impose this constraint to perform comparisons between different portfolio strategies without the need of defining a risk-aversion parameter. My approach though can be easily modified to allow investment in the risk-free asset.

It is intuitive to express the optimal solution of the problem (1), (2), (3) as a linear combination of two special portfolios. The first results if I do not impose the constraint (2). In this case, the investor should hold the global minimum variance portfolio, which is the portfolio of risky assets with minimum risk. The weights are defined as:

$$
w^{\mathrm{MIN}}=\frac{\Sigma^{-1} 1_{N}}{1_{N}^{\prime} \Sigma^{-1} 1_{N}} .
$$


The second portfolio is defined as the point where the capital Market Line is tangent to the efficient frontier. For this reason, it is called tangency portfolio and its weights are given by

$$
w_{t}^{\mathrm{TP}}=\frac{\Sigma^{-1} \mu}{1_{N}^{\prime} \Sigma^{-1} \mu} \text {. }
$$

Then, applying first order conditions to the mean-variance problems confirms that the solution $w^{\mathrm{MV}}$ as well as every point in the frontier is a linear combination of the global minimum variance portfolio and the tangency portfolio

$$
w^{\mathrm{MV}}=\left(1-x_{t}\right) w^{\mathrm{MIN}}+x_{t} w^{\mathrm{TP}}
$$

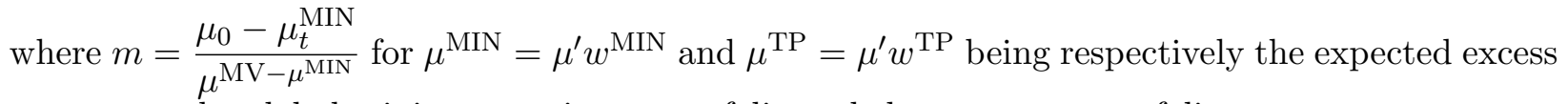
returns on the global minimum variance portfolio and the tangency portfolio.

If short-sales are not allowed, there is one additional constraint in the optimization problem to ensure the positivity of the weights:

$$
w \geq 0
$$

The short-sale constrained optimization problem cannot be solved analytically, but there are fast quadratic optimization algorithms to derive the optimal weights.

\section{$3 \quad$ Estimation risk and portfolio stability}

All efficient portfolios as well as their short-constrained counterparts are functions of the first two moments $\mu$ and $\Sigma$ of the excess returns. In practice, $\mu$ and $\Sigma$ are unknown and the investor needs to estimate them. This estimation is usually performed using a sample of the history of the asset excess returns $r_{t-T} \ldots . r_{t-1}$ where $T$ is sample size. For example, the traditional practice employs the Maximum Likelihood (ML) estimators:

$$
\begin{gathered}
\hat{\mu}_{t}=\frac{1}{T} \sum_{i=t-T}^{t-1} R_{i} \\
\hat{\Sigma}_{t}=\frac{1}{T} \sum_{i=t-T}^{t-1}\left(R_{i}-\hat{\mu}_{t}\right)\left(R_{i}-\hat{\mu}_{t}\right)^{\prime}
\end{gathered}
$$

and applies them to estimate the portfolio weights. Using estimates instead of the true values of the parameters introduces estimation risk in the portfolio choice process. Estimation risk is known to result in severely suboptimal portfolios which produce low or even negative risk-adjusted returns (see for instance, Michaud, 1989; Best and Grauer, 1991). A vast literature over the last decades proposes several alternative estimators for the moments or the weights that result in improved performance (Brandt, 2009 offers an excellent review). The majority of the proposed strategies, however, performs worse than naive diversification (DeMiguel et al., 2009a, DGU hereafter) in terms 
of out-of-sample risk adjusted returns. In response to this finding, new studies have developed more efficient portfolio strategies that produce higher risk-adjusted returns (DeMiguel et al. 2009b, Tu and Zhou, 2010, Kirby and Ostdiek, 2012, Kourtis et al. 2012).

The effects of estimation risk are more prominent in the presence of transaction costs since estimation errors lead to unstable portfolios over time. The standard measure of instability is portfolios turnover. The turnover measures the deviation of the portfolio weights after rebalancing from the weights before rebalancing using the 1-norm. For a sample-based strategy $p$, the turnover at period $t$ is given by

$$
\tau_{t}^{p}=\left\|\hat{w}_{t}^{p}-\tilde{w}_{t}^{p}\right\|_{1}
$$

where $\tilde{w}_{t}^{p}$ are the portfolio weights for the strategy at time $t$ before rebalancing. These are defined as

$$
\tilde{w}_{i, t}^{p}=\frac{\hat{w}_{i, t-1}^{p}\left(1+R_{i, t-1}\right)}{1+\sum_{i=1}^{N} \hat{w}_{i, t-1}^{p} R_{i, t-1}} .
$$

To understand how estimation risk affects stability, note that the turnover of asset $i$ is

$$
\left|\hat{w}_{i, t}^{p}-\tilde{w}_{i, t}^{p}\right|=\left|\left(\hat{w}_{i, t}^{p}-\hat{w}_{i, t-1}^{p}\right)-\frac{\hat{w}_{i, t-1}^{p}\left(R_{i, t}-R_{t}^{p}\right)}{\left(1+R_{t}^{p}\right)}\right|
$$

where $R_{t}^{p}$ is the return on the strategy $p$ at time $t$. The above equation implies that compared to a constant-weights strategy such as $1 / N$ or the optimal true portfolio, the turnover of an asset

for a sample-based strategy may be larger by $\left|\left(\hat{w}_{i, t}^{p}-\hat{w}_{i, t-1}^{p}\right)\right|$. Sample-based strategies with a large sensitivity in changes in the sample may see a large decline in the returns in the presence of transaction costs. I examine whether this argument is valid for several sample strategies in the literature that I next discuss. Table 1 lists all strategies employed in this work.

\subsection{Naive diversification $(1 / N)$}

I start with naive diversification which is the benchmark strategy in this paper. Naive diversification assigns the same weight to each asset:

$$
w_{t}^{1 / N}=\frac{1_{N}}{N}
$$

Even though it is a biased estimator of the optimal portfolio weights, it is not subject to estimation risk and this explains its favorable performance reported by DeMiguel et al. (2009a) compared to several strategies in the literature. Other attractive features of this strategy include the positivity of weights and the low turnover due to the constant weights.

\subsection{The sample tangency portfolio (TP)}

The sample tangency portfolio results from replacing $\mu$ and $\Sigma$ with $\hat{\mu}$ and $\hat{\Sigma}$ in (5). The portfolio weights are then given by 


$$
\hat{w}_{t}^{\mathrm{TP}}=\frac{\hat{\Sigma}_{t}^{-1} \hat{\mu}_{t}}{1_{N}^{\prime} \hat{\Sigma}_{t}^{-1} \hat{\mu}_{t}} .
$$

This portfolio has the worst performance in DGU producing highly negative Sharpe-ratios in several cases and very high levels of turnover. Kirby and Ostdiek (2012) attribute this performance to the denominator of in the weights. If $1_{N}^{\prime} \Sigma^{-1} \mu$ is close to zero, the sample tangency portfolio will have extreme weights leading to severe out-of-sample performance. This is because the required return return $\mu^{\prime} \hat{w}_{t}^{\mathrm{MV}}$ is unrealistically high and this magnifies both the turnover and the estimation risk.

\subsection{The sample global minimum variance portfolio (MIN)}

The means $\mu$ are considered more challenging to estimate than variance-covariances (Merton, 1980; Chopra and Ziemba, 1993). Motivated by this, several authors propose the use of the sample global minimum variance portfolio defined in (4) (Jagannathan and Ma, 2003, Ledoit and Wolf, 2003, DeMiguel et al. 2009b, Kourtis et al. 2012, among others)

$$
\hat{w}_{t}^{\mathrm{MIN}}=\frac{\hat{\Sigma}_{t}^{-1} 1_{N}}{1_{N}^{\prime} \hat{\Sigma}_{t}^{-1} 1_{N}}
$$

Jagannathan and Ma (2003) and DGU find that the above portfolio outperforms the sample tangency portfolio in terms of Sharpe ratios and certainty-equivalent returns in several datasets of real returns. Recently, Kourtis et al. (2012) also derive in closed form the expected certaintyequivalent return of the above strategy and analytically confirm the empirical results of previous studies. GMV is also more stable than the tangency portfolio over time since the denominator of the weights is always positive due to the positive definiteness of $\hat{\Sigma}_{t}$. As a results, it does not attain values close to zero.

\subsection{The sample mean-variance portfolio (MV, MVC)}

The sample-based mean-variance portfolio is the sample counterpart of (6):

$$
\hat{w}_{t}^{\mathrm{MV}}=\left(1-\hat{x}_{t}\right) \hat{w}_{t}^{\mathrm{MIN}}+\hat{x}_{t} \hat{w}_{t}^{\mathrm{TP}}
$$

where $\hat{m}_{t}$ results for using the ML estimators of the parameters. The above equation defines a " 2 fund" strategy, since it is a weighted -sum of the sample tangency portfolio and the global minimum variance portfolio. Then, the variation of level of expected return $\mu_{0}$ defines the sample minimum frontier. For $0 \leq \hat{x}_{t}<1, \hat{w}_{t}^{\mathrm{MV}}$ is less sensitive to estimation risk and more stable than the sample tangency portfolio. This is because decreasing $\hat{x}_{t}$, increases the weight given to the global minimum variance portfolio.

For my comparisons, I also need to set $\hat{x}_{t}$. Equivalently, I need to pick an appropriate value for

$\mu_{t}^{0}$. Following Kirby and Ostdiek (2012), I choose $\mu_{t}^{0}$ to be equal the sample mean return of $1 / N$ 
unless the latter portfolio is not in the efficient part of the sample minimum variance frontier. In this case, I set the required return equal to the return of the closest efficient portfolio which is the global minimum variance portfolio. In essence:

$$
\mu_{t}^{0}=\max \left\{\mu^{1 / N}, \mu^{\mathrm{MIN}}\right\}
$$

where $\mu^{1 / N}=\hat{\mu}^{\prime} 1_{N} / N$.

This allows me to fairly compare the performance of the mean-variance portfolio to that of $1 / N$. Moreover, as shown by Kirby and Ostdiek (2012), this setting produces superior portfolios compared to the tangency strategy in terms of both stability and out-of-sample performance. I additionally consider the short-sale constrained version of this strategy denoted by (MVC).

\subsection{The Ledoit-Wolf minimum variance portfolio (LW)}

All sample-based portfolios so far employ the ML estimators of $\mu$ and $\Sigma$. Ledoit and Wolf (2003, 2004a, 2004b) develop an improved estimator of the covariance matrix based on the shrinkage methodology of James and Stein (1961). The estimator is a convex combination of $\hat{\Sigma}$ and a matrix $\hat{\Lambda}_{t}$ that is less prone to estimation errors:

$$
\hat{\Sigma}_{t}^{\mathrm{LW}}=\left(1-\hat{f}_{t}\right) \hat{\Sigma}+\hat{f}_{t} \hat{\Lambda}_{t}
$$

The set of candidates for $\hat{\Lambda}$ include the identity matrix (Ledoit and Wolf 2004b), the constant correlations matrix (Ledoit and Wolf 2004a) and the covariance matrix that results from the CAPM model (Ledoit and Wolf 2003). A statistical loss function is employed for the optimal computation of $\hat{f}_{t}$. Then, the global mimimum variance weights can be estimated by

$$
\hat{w}_{t}^{\mathrm{LW}}=\frac{\left(\hat{\Sigma}_{t}^{\mathrm{LW}}\right)^{-1} 1_{N}}{1_{N}^{\prime}\left(\hat{\Sigma}_{t}^{\mathrm{LW}}\right)^{-1} 1_{N}}
$$

Ledoit and Wolf $(2003 ; 2004 a)$ find that the above strategy outperforms GMV in many cases with regards to out-of-sample variance and Sharper. In this paper, I only consider $\Lambda_{t}=I$ as a shrinkage target where $I$ is the identity matrix. This is because I find that it produces the lowest out-of-sample variance compared to using the constant-correlations matrix and the matrix from an 1-factor returns model.

\subsection{The 2-norm-constrained portfolio (NC)}

Portfolio constraints such as no-short-sales are known to improve performance (Jagannathan and Ma, 2003). In this context, Demiguel et al. (2009b) show that norm-constrained minimum variance portfolios can outperform $1 / N$ out-of-sample. They specifically augment (1),(3) with the following constraint 


$$
\|w\| \leq \delta_{t}
$$

where $\|\cdot\|$ can be the 1-norm, 2-norm or a matrix norm. They use two criteria to select the parameter $\delta_{t}$. The first involves a cross-validation method for computing the parameter that minimizes out-ofsample variance. The second chooses the value of $\delta$ that maximises the last period's return. In this manner, it aims to increase out-of-sample returns by taking into account the positive autocorrelation in portfolio returns (see Campbell et al, 1997). In my analysis, I only include the 2-norm constrained portfolio (NC) that maximises last period's return on the basis of two reasons. First, the variancebased portfolios in DeMiguel et al. (2009b) appear to perform worse than LW portfolio with regards to out-of-sample variance. Second, the 2-norm portfolio results in higher or similar Sharpe ratios than portfolios based on other norms.

\subsection{The three-fund strategy of Tu and Zhou (2011) (3F)}

Under the assumption of normal i.i.d. returns, Tu and Zhou (2011) propose the following strategy;

$$
\hat{w}_{t}^{3 \mathrm{~F}}=\pi_{1} \hat{w}_{t}^{\mathrm{TP}}+\pi_{2} \hat{w}_{t}^{\mathrm{MIN}}+\pi_{3} \hat{w}_{t}^{1 / N}
$$

where $\pi_{i}$ 's are chosen to maximize expected certainty-equivalent return under normality. Tu and Zhou (2011) find that this portfolio produces higher risk-adjusted returns that $1 / N$.

The weights in (21) define a four-fund strategy since it combines the TP, MIN, $1 / N$ and the riskfree asset ( $c_{i} s$ do not sum to unity). In order to make the performance of this strategy independent of the risk-free asset and comparable to the remaining strategies in my analysis, I divide the weights by $1_{N}^{\prime} \hat{w}_{t}^{3 \mathrm{~F}}$ to end up with a portfolio of risky assets only. ${ }^{1}$

\subsection{The volatility timing strategy (VT)}

Kirby and Ostdiek (2012) propose several strategies that mimic some of the attractive features of $1 / N$ while they take into account the information contained in historical returns. They show that these timing strategies outperform $1 / N$ out-of-sample no matter the presence of transaction costs. Among the different timing strategies they propose, I consider here the following:

$$
\hat{w}_{t}^{\mathrm{VT}}=\frac{\hat{D}_{t} 1_{N}}{1_{N}^{\prime} \hat{D}_{t} 1_{N}},
$$

where $D_{t}$ is the diagonal covariance matrix, i.e. a matrix with the asset variances in the main diagonal and zeros elsewhere. This strategy is particularly attractive since it offers a very low turnover and positive weights. Kirby and Ostdiek (2012) also find that it offers a significantly higher Sharpe ratio than $1 / N$.

\footnotetext{
${ }^{1}$ This normalization would not be plausible if $1_{N}^{\prime} \hat{w}_{t}^{3 \mathrm{~F}}$ is taking values close to 0 such as the tangency portfolio. I find that this is not the case in the datasets I consider due to the form of the weights (21). Moreover, I find that the performance when investing in the risk-free asset is slightly worse.
} 


\subsection{Portfolios based on the shrinkage of the inverse covariance matrix (ICV, ICR)}

The two remaining strategies I include in my analysis are derived by Kourtis et al. (2012) who propose to apply the shrinkage methodology directly to the inverse covariance matrix. Kourtis et al. (2012) develop the following estimator:

$$
\hat{S}_{t}=\hat{d}_{t} \hat{\Sigma}_{t}^{-1}+\left(1-\hat{d}_{t}\right) \hat{F}_{T}
$$

where $F$ is a matrix less sensitive to estimation errors than $\hat{\Sigma}^{-1}$. They apply $\hat{S}$ to estimate the global minimum variance portfolio:

$$
\hat{w}_{t}^{\text {ic }}=\frac{\left(\hat{S}_{t}\right)^{-1} 1_{N}}{1_{N}^{\prime}\left(\hat{S}_{t}\right)^{-1} 1_{N}}
$$

Kourtis et al. (2012) select $\hat{d}_{t}$ in two ways similar to DeMiguel et al. (2009b); the first uses the cross-validation technique to reduce out-of-sample variance and the second maximizes last period's return. They find that the first approach offers very low levels of risk while the second results in higher Sharpe Ratios compared to other strategies from the literature (including $1 / N$ ). Among different candidates for $\hat{F}$, Kourtis et al. (2012) find that a linear combination of the identity matrix and the covariance matrix for the market model of Sharpe (1963) gives the best performance. Hence, I only consider this matrix while construction $\hat{w}_{t}^{\mathrm{IC}} . I C V$ and $I C R$ respectively denote the minimum variance portfolio that results from choosing $\hat{d}_{t}$ according to the variance and the return approach.

\subsection{Performance and stability of sample-based portfolios}

I now show that most of the sample-based strategies presented can be unstable over time and perform worse than $1 / N$ in the presence of transaction costs. For this purpose, I employ 5 datasets of real monthly excess return that include 3 datasets that have been used in several previous studies. These are the three Fama-French factors (3FF), the 10 industry portfolios (10Ind) and the 25 size and book-to-market portfolios (25SBM). The fourth dataset (8int) consists of US-dollar denominated returns on 8 international market portfolios (Australia, Canada, France, Germany, Japan, Italy, UK, USA). Finally, I consider a dataset of 50 plain stocks (50SP). These are the current S\&P500 stocks that have been traded since 01/1981. As a risk-free rate, I adopt the 1month T-bill rate. All datasets are obtained from Kenneth French's website except for the stocks dataset which is obtained by Google. Table 3 includes a summary of the datasets along with the time period each one spans. The selection of datasets allows to evaluate portfolio performance for different numbers and types of assets at both national and international level.

In each dataset, I study how the ten strategies perform in terms of out-of-sample risk-adjusted returns and stability over time using the rolling window method of DeMiguel et al. (2009a). In particular, for each month $t$, I use the returns for the months $t-T, \ldots, t-1$ to compute the portfolio 
weights $\hat{w}_{t}^{p}$ for each strategy $p$. In this manner, I obtain a time series of monthly excess returns $\left\{r_{t}^{p}=r_{t}^{\prime} \hat{w}_{t}^{p}\right\}$ for each strategy and dataset. I compute the mean $\hat{\mu}_{p}$ and variance $\left(\hat{\sigma}^{p}\right)^{2}$ of these returns as well as the Sharpe ratio:

$$
\hat{\lambda}^{p}=\frac{\hat{\mu}^{p}}{\hat{\sigma}^{p}}
$$

which is the standard measure of the efficiency of a portfolio strategy. I also study the significance of the difference between the Sharpe ratio of each sample-based strategy and $1 / N$. For this purpose, I test the hypothesis " $\mathrm{H}_{0}: \widehat{S R}_{p}-\widehat{S R}_{1 / N}=0$ " following the studentised circular block bootstrap approach of Ledoit and Wolf (2008) to obtain the respective p-value for the difference.

I characterize the stability of a strategy through the average turnover $\hat{\tau}^{p}=$ $\frac{1}{M-T-1} \sum_{t=T+1}^{M-1} \tau_{t}^{p}$. High average turnover is associated with unstable portfolios and high transaction costs. In this paper, I account for proportional variable transaction costs. Then, the portfolio return net of transaction costs $\tilde{R}_{t}^{p}$ is given by

$$
\tilde{R}_{t}^{p}=\left(1+R_{t}^{p}\right)\left(1-\kappa \tau_{t}^{p}\right)-1
$$

where $\kappa$ stands for the level of transaction costs per transaction and $\tau_{t}^{p}$ is the turnover at time $t$.

In Panel A of Tables 3-7 I present the mean, variance, Sharpe ratio in annual terms and the turnover for each portfolio strategy in the absence of transaction costs as well as the Sharpe ratio in the presence of transaction costs of 100 basis points $(\kappa=0.01)$. One, two and three '*' reflect significance at $10 \%, 5 \%$ and $1 \%$, respectively. I compute these performance measures for a sample size of 120 months (10 years of monthly returns). Since the computation of the portfolio weights of the 3 -fund strategy requires a risk aversion parameter, I set it equal to $3 .^{2}$

When there are no transaction costs, the results are consistent with the existing literature even though $1 / N$ manages to outperform most strategies in the 8Int and 50SP datasets which are only used here. With regards to portfolio risk, the strategies based on the shrinkage estimator of the covariance matrix and its inverse (LW and ICV, respectively) offer lower variances compared to both $1 / N$ and the global minimum variance portfolio (MIN).

The results for the Sharpe ratios show the tangency portfolio is the worst performer in line with the literature. It offers higher Sharpe ratio than $1 / N$ only for the the $3 \mathrm{FF}$ and $25 \mathrm{SBM}$ datasets while it even results in negative average return for the 50SP set. The Sharpe ratio for the meanvariance portfolio with an expected return equal to than of the $1 / N$ portfolio is higher than that of the $1 / N$ portfolio and the global minimum variance portfolio in all datasets except for the set of 50 stocks. The latter is the only set where the short-sale constraint improves performance. The five portfolios from the recent literature (NC, 3F, VT, ICV, ICR) outperform $1 / N$ in the 10IND and $25 \mathrm{SBM}$ portfolios in line with previous studies. In the $3 \mathrm{FF}$ set, the norm-constrained portfolio and the 3-fund rule of $\mathrm{Tu}$ and Zhou (2012) offer the highest Sharpe ratio. In the international dataset, MV, MVC, VT and ICV outperform $1 / N$. The most challenging dataset is the 50SP dataset where only the norm-constrained portfolio and the volatility timing strategy outperform

\footnotetext{
${ }^{2}$ I have also considered alternative values for the risk aversion parameter with no significant change in the results
} 
$1 / N$. These results highlight the need of more efficient portfolios.

I now show the importance of portfolio stability. All sample-based strategies except for the volatility-timing portfolio produce significantly higher turnover than $1 / N$. As a result, the Sharpe ratios net of transaction costs are significantly lower for all strategies except for VT. The reduction is quite prominent for the recent contributions in the literature (NC, 3F, ICR). In fact, the Sharpe ratios for these three strategies are negative in most of the datasets. In other words, the instability of portfolio strategies can result in significant losses in practice. Motivated by this findings, I develop an approach to improve portfolio stability.

\section{A stability penalty to mean-variance optimization}

The discussion in the previous section highlights the need of efficient portfolio strategies that offer low turnover. The natural approach followed in the literature is to modify the constraint (2) by subtracting the proportional transaction costs from the expected portfolio return:

$$
w_{t}^{\prime} \mu-c\left\|w_{t}-\tilde{w}_{t}\right\|_{1} \geq \mu^{0}
$$

In contrast to the original mean-variance problem, the transaction costs-adjusted problem involves a nonlinear constraint. As such, the derivation of an analytic representation of the optimal weights is not feasible and their calculation may be computationally demanding for a large number of assets. It is also uncertain whether the constraint (27) improves performance significantly. For this reason, I study the out-of-sample performance of the transaction costs adjusted mean-variance portfolio and its short-sale constrained counterpart. I use the "fmincon" function of Matlab to derive the portfolio weights. I again assume of proportional transaction costs of $100 \mathrm{bp}$. I present the results in Table 8. Clearly, both sample-based portfolios are more efficient and stable compared to the original portfolios. However, in two out of the five datasets $1 / N$ still outperforms the mean-variance portfolios in terms of Sharpe ratio. This indicates the need for more efficient strategies. However, the lack of an analytical representation of the optimal weights under transaction costs does not allow the application of most of the recent advances in the estimation risk literature. For instance, the strategies of Kourtis et al. and Tu and Zhou use the analytical form of the optimal weights. DeMiguel et al. (2012) also found that adding the non linear constraint to the norm-constrained portfolio worsens performance. They argue that this is due to the fact the constraint involves the difference between a term that involves the unknown $\mu$ and a term that is free of estimation errors.

I resolve these issues by proposing an alternative method to produce stable portfolios. I introduce a penalty directly in the objective function instead of the expected return as:

$$
\min _{w_{t}} w_{t}^{\prime} \Sigma w_{t}+c_{t}\left(w_{t}-\tilde{w}_{t}\right)^{\prime} \Sigma\left(w_{t}-\tilde{w}_{t}\right)
$$

Similarly to transaction costs, the penalty increases as the weights move away from the current 
holdings $\tilde{w}_{t}$. However, in contrast to using 1-norm, the deviation is measured by the $\Sigma^{\frac{1}{2}}$ norm. Note that the objective function can be rewritten as

$$
\left\|w-w^{\mathrm{MIN}}\right\|_{\Sigma^{\frac{1}{2}}}^{2}+c_{t}\|w-\tilde{w}\|_{\Sigma^{\frac{1}{2}}}^{2}
$$

In this manner, the objective function now represents a trade-off between efficiency and instability. The above modification of the mean-variance problem offers two advantages compared to other methods in the literature that incorporate transaction costs. First, all terms in the objective function are now subject to estimation risk. This may help to mitigate the problem discussed in DeMiguel et al. (2012). Second, I am now able to derive the optimal portfolio weights in closed form as the next proposition implies.

Proposition 1 The solution to the new portfolio choice problem is given by

$$
\bar{w}_{t}^{M V}=\frac{1}{1+c_{t}} w_{t}^{M V}+\frac{c_{t}}{1+c_{t}} \tilde{w}_{t}
$$

Proof. The first order conditions for the stable portfolio choice problem are:

$$
2\left(1+c_{t}\right) \Sigma w_{t}-\delta_{1} 1_{N}-\delta_{2} \mu+2 c_{t} \Sigma \tilde{w}_{t}
$$

and solving for $w$ gives:

$$
w_{t}=\frac{\delta_{1}}{2\left(1+c_{t}\right)} \Sigma^{-1} 1_{N}+\frac{\delta_{2}}{2\left(1+c_{t}\right)} \Sigma^{-1} \mu+\frac{c_{t}}{\left(1+c_{t}\right)} \tilde{w}_{t}
$$

Applying the constraints (2), (3) results to a linear system. If I then solve for $\delta_{1}$ and $\delta_{2}$, I end up with (30).

Therefore, the optimal portfolio is a linear combination of the optimal portfolio without the penalty and the current holdings of the investor. This holds for all portfolios in the mean-variance frontier including the global minimum variance portfolio.

\subsection{Treating estimation risk}

The analytical form of the portfolio weights allows to jointly treat estimation risk and instability. This is because one can use any portfolio strategy from the literature to estimate the original mean variance weights $w_{t}^{\mathrm{MV}}$ in (30). Then, for any strategy $p$, there is a stable counterpart with weights given by

$$
\bar{w}^{p}=\frac{1}{1+c_{t}^{p}} \hat{w}_{t}^{p}+\frac{c_{t}^{p}}{1+c_{t}^{p}} \tilde{w}_{t}^{p}
$$

Moreover, there may be further portfolio performance improvements due to the imposition of the stability penalty. To better understand this argument, I provide two interpretations of the 
stability penalty. First, note that $c_{t}^{p}$ is the Lagrange multiplier of the constraint

$$
\mid w-\tilde{w} \|_{\Sigma^{\frac{1}{2}}}^{2}<\delta
$$

for some $\delta$ when this constraint is added in the original problem. DeMiguel et al. (2009b) show that imposing norm constraints may improve portfolio performance in the presence of estimation risk. Therefore, strategies which are subject to significant estimation errors may benefit from the stability penalty even in the absence of transaction costs.

The second interpretation relies on the shrinkage estimation theory developed by James and Stein (1961). In this case, the stable portfolio weights $\bar{w}^{p}$ result from shrinking the original portfolio weights $\hat{w}_{t}^{p}$ to the weights of the buy-and-hold strategy $\tilde{w}_{t}^{p}$. On one hand, the original portfolio is a contrarian strategy; it performs well when the serial correlation of the returns is negative. On the other hand, the buy-and-hold strategy is a momentum strategy offering high returns when the asset returns are positively autocorrelated. Therefore, the stable portfolio diversifies between a contrarian and a momentum strategy and this may lead to performance improvements when the market is in momentum.

\subsection{Treating portfolio instability}

I now discuss how the stability penalty reduces the magnitude of transaction costs for any strategy $p$. It is simple to verify that the turnover of the stable portfolio is

$$
\bar{\tau}_{t}^{p}=\frac{1}{1+c_{t}^{p}} \hat{\tau}_{t}^{p}
$$

As the above relation indicates, for $c_{t}^{p} \geq 0$ the turnover of the stable portfolio is decreasing with $c_{t}^{p}$ and is less than the turnover of the original portfolio. The larger $c_{t}^{p}$ is, the more stable the portfolio is and the less the transaction costs are. In the special case that $c_{t}^{p}$ goes to infinity, the investor will select the portfolio of the previous period and transaction costs will approach 0 .

The choice of the stability parameter $c_{t}^{p}$ is critical in my approach. In this work, I propose two alternative ways to choose it. ${ }^{3}$ First, at each period $t$, I set the value of $c_{t}^{p}$ that matches the turnover of the stable strategy to that of $1 / N$. This is because $1 / N$ is known to offer very low turnover compared to other strategies while being the benchmark in this paper. Then, the stability parameter is given by

$$
\hat{c}_{t}^{p}=\frac{\hat{\tau}_{t}^{p}-\hat{\tau}_{t}^{1 / N}}{\hat{\tau}_{t}^{1 / N}}
$$

so that $\bar{\tau}_{t}^{p}=\hat{\tau}_{t}^{1 / N}$.

To further improve performance, the second approach for selecting the stability parameter makes use of the positive autocorrelation of portfolio returns in line with the work of Demiguel

\footnotetext{
${ }^{3}$ Alternative to these, one can easily fix the stability parameter in order to achieve a desired level of transaction costs.
} 
et al. (2009b) and Kourtis et al. (2012). In particular, I choose the value of $c_{t}^{p}$ that lies in the interval $\left[0, \hat{c}_{t}^{p}\right]$ so that the resulting stable portfolio maximizes the return of the previous period. As a result, at each period, the investor will either hold the stable portfolio for $c_{t}^{p}=\hat{c}_{t}^{p}$ or she will not trade. In the latter case, the turnover will be zero further reducing the average turnover and transaction costs over time.

In the following, I present an efficient method for estimating the expected turnover of $1 / N$. The latter is central in my approach since it characterizes the stability of all stable portfolios and the magnitude of transaction costs that one should expect.

\subsection{The distribution of the turnover}

Several studies investigate the distribution of the portfolio weights, means, variance, certaintyequivalent return and the Sharpe ratio (e.g., Ohkrin and Schmid, 2006, Kan and Zhou, 2007, Kan and Smith, 2008, Kourtis et al. 2012). In light of the results of this study, portfolio turnover is also an important variable in portfolio optimization. It is important then to study the distribution of the portfolio turnover. Assuming normality, I next present an analytic approximate expression for the distribution and the expectation turnover of the $1 / N$ strategy. This allows to make inference on the expected turnover of the stable portfolios proposed in this paper. Let $R_{t} \sim N\left(m_{t}, \Sigma\right)$ where $m_{t}=\mu+r_{t}^{f}$. The turnover of $1 / N$ at time $t$ is

$$
\tau_{t}^{1 / N}=\sum_{i=1}^{N}\left|\frac{1}{N}-\frac{\frac{1}{N}+R_{i, t}}{1+\sum_{j=1}^{N} \frac{R_{j, t}}{N}}\right|=\sum_{i=1}^{N}\left|\frac{\bar{R}_{t}-R_{j, t}}{N\left(1+\bar{R}_{t}\right)}\right|
$$

where $\bar{R}_{t}=\sum_{j=1}^{N} \frac{R_{j, t}}{N}$ is the cross-sectional average of the returns. To determine the distribution of $\tau_{t}^{1 / N}$, I first examine the joint distributions of the numerator and denominator by setting $Y_{i, t}=$ $\bar{R}_{t}-R_{t}^{i}$ and $X_{t}=N\left(1+\bar{R}_{t}\right)$. It is easy to confirm that $Y_{i, t}$ and $X_{t}$ are normally distributed as sums of normally distributed variables. Their first two moments and their covariance are given by

$$
\begin{array}{r}
\mu_{i, t}^{Y}:=E\left(Y_{i, t}\right)=\bar{m}_{t}-m_{i, t} \\
\left(\sigma_{i, t}^{Y}\right)^{2}=\frac{1_{N}^{\prime} \Sigma 1_{N}}{N^{2}}+\sigma_{i i, t}^{2}-2 \frac{e_{i}^{\prime} \Sigma 1_{N}}{N} \\
\mu^{X}:=E\left(X_{t}\right)=N\left(1+\bar{m}_{t}\right) \\
\left(\sigma_{t}^{X}\right)^{2}=1_{N}^{\prime} \Sigma 1_{N} \\
\sigma^{Y_{i, t}, X_{t}}=\frac{1_{N}^{\prime} \Sigma 1_{N}}{N}-\frac{e_{i}^{\prime} \Sigma 1_{N}}{N}
\end{array}
$$

for $e_{i}$ being a $N$-dimensional vector with 1 in the $i-t h$ entry and zero elsewhere. The exact distribution of the ratio of two dependent normal variables $(Y, X)$ is the subject in several studies (e.g., Marsaglia, 1965, Hinkley 1969). In general, the moments of the ratio do not exist. Under the assumption of $P\left(X_{t}{ }^{6} 0 \simeq 0\right)$, Hayya et al. (1975) provide a handful approximation of the distribution $Y / X$ that I employ in this paper. Hayya et al. show that $Y / X$ approximately follows a normal 
distribution if

$$
c v(X)<0.09, c v(Y)>0.19,|\rho(Y, X)|<0.5
$$

where $c v$ and $\rho$ respectively denote the coefficient of variation and the correlation. Table 9 reports $c v\left(X_{t}\right)$ and the cross-sectional average, minimum and maximum of $c v\left(Y_{i, t}\right)$ and $\left|r h o\left(Y_{i, t}, X_{t}\right)\right|$ for each dataset of returns estimating $m_{t}$ and $\Sigma_{t}$ with the sample mean and covariance matrix. I observe that the conditions (43) are fulfilled. Therefore, an approximation of the distribution of $W_{i, t}=\frac{Y_{i, t}}{X_{t}}$ can be obtained through Taylor approximation as

$$
\begin{array}{r}
W_{i, t} \sim N\left(\mu_{t}^{W_{i}},\left(\sigma^{W_{i}}\right)_{t}^{2}\right) \\
\mu_{t}^{W_{i}}=\frac{\mu^{Y_{i, t}}}{\mu^{X}}+\left(\sigma_{t}^{X}\right)^{2} \frac{\mu_{i, t}^{Y}}{\left(\mu_{t}^{X}\right)^{3}}-\frac{\sigma^{Y_{i, t}, X_{t}}}{\left(\mu_{t}^{X}\right)^{2}} \\
\left.\left(\sigma_{t}^{W_{i}}\right)^{2}=\left(\sigma_{t}^{X}\right)^{2}\right) \frac{\left(\mu_{i, t}^{Y}\right)^{2}}{\left(\mu_{t}^{X}\right)^{4}}+\frac{\left(\sigma_{i, t}^{Y}\right)^{2}}{\left(\mu_{t}^{X}\right)^{2}}-2 \frac{\sigma^{Y_{i, t}, X_{t}} \mu_{i, t}^{Y}}{\left(\mu_{t}^{X}\right)^{3}}
\end{array}
$$

Given the approximate normality of $W_{i, t}$, the turnover of each asset $\left|W_{i, t}\right|$ approximately follows a folded normal distribution with the same distribution parameters. In figure 1, I compare the real cdf of the turnover of the first asset (solid line) to the approximate cdf (dashed line) for the 8Int, 10ind, 25SBM and 50SP datasets. The cdfs are computed using a sample of 10,000 simulated returns drawn from the multivariate normal distribution with moments equal to the sample moments of the four datasets. The figure confirms that the approximation is accurate in all datasets. Therefore, the expected turnover for the $i$-asset of the $1 / N$ strategy can be computed by

$$
E\left|W_{i, t}\right|=\sigma_{t}^{W_{i}} \sqrt{\frac{2}{\pi}} e^{-\frac{\left(\mu_{t}^{W_{i}}\right)^{2}}{2\left(\sigma_{t}^{W_{i}}\right)^{2}}}+\mu_{t}^{W_{i}}\left(\left(1-2 \Phi\left(-\frac{\mu_{t}^{W_{i}}}{\sigma_{t}^{W_{i}}}\right)\right)\right.
$$

where $\Phi$ is the cdf of the standard normal distribution.

The distribution of the turnover of the $1 / N$ strategy can then be approximated by a sum of folded normal distributions $\left(W \sim \sum_{i=i}^{N} F N\left(\mu^{W_{i}},\left(\Sigma^{W}\right)^{2}\right)\right)$. An approximation of the expected turnover is then given

$$
E\left(\hat{\tau}^{1 / N}\right)=\sum_{i=1}^{N} E\left|W_{i, t}\right|
$$

The above approximation allows the estimation of the turnover of $1 / N$ using the ML estimators of the sample moments. In panel B of Table $9 \mathrm{I}$ compare the average of this estimator to the average realised turnover of the $1 / N$ strategy (Tables $3-7$ ). The estimation is performed using a rolling window approach with a sample size of 120 months. The results confirm the accuracy of the estimator which also offers low standard errors. 


\section{$5 \quad$ Performance of the stable portfolios}

In this section, I evaluate the stability approach by applying it to the sample-based strategies presented in section 3. Panel B of Tables 3-7, contains the mean, variance and turnover of the stable strategies as well as their Sharpe ratio in the absence and presence of transaction costs. The first column for the turnover and the transaction costs-adjusted Sharpe ratio corresponds to the stability parameter that matches the turnover of the sample-based strategy to that of $1 / N$. The second column reports in parenthesis these measures when the stability parameter maximizes last period's return. All performance metrics are derived following the procedure discussed in Panel A. It is convenient to discuss the results in three parts since there are similarities between some datasets.

\subsection{Performance in the 3FF dataset}

This dataset contains the returns of only 3 assets leaving less room for estimation risk. As the results in Panel A of Table 3 indicate, $1 / N$ achieves a Sharpe ratio of 0.841 when there are no transaction costs while the norm-based portfolio and three-fund strategy produce the highest Sharpe ratios of 0.96 and 0.946 respectively. In fact, most portfolio strategies perform better than naive diversification. Interestingly though, $1 / N$ outperforms the minimum variance portfolio (MIN) and the shrinkage-based portfolios (LW, ICV, and ICR) as well as the volatility timing strategy.

Due to the small number of assets, the level of turnover for the sample-based strategies is comparatively low in comparison with the remaining datasets. As expected, $1 / N$ is the most stable strategy with a turnover of 0.023 . The remaining strategies attain similar levels excluding $\mathrm{NC}$ and ICR which result in turnover of 0.161 and 0.411 , respectively. Because of the large turnover of the latter strategy, the corresponding Sharpe ratio is negative once I account for transaction costs of $100 \mathrm{bp}$. This result indicates the importance of portfolio stability.

Examining the results in Panel B, I find that the stability penalty improves the Sharpe ratio of almost all strategies, even in the absence of transaction costs. The most significant improvement is observed for the ICR strategy where the Sharpe ratio increases from 0.666 to 0.909 higher than that of $1 / N$. For most strategies, the increase in the Sharpe ratio mainly comes from an increase in the mean return rather than a decrease in the variance. When the stability parameter is chosen to maximize portfolio return, the turnover ranges from 0.006 to 0.016 significantly lower than the turnover of $1 / N$.

The improvement in the performance due to the stability approach is more prominent in the presence of transaction costs. More notably, the Sharpe ratio of the norm-based and the ICR portfolios respectively increase from 0.626 and -0.052 to 0.84 and 0.866 when the turnover is equal to that of $1 / N$. When the stability parameter is selected according to the momentum criterion, portfolio performance is further improved to the point that all sample-based strategies apart from the volatility timing strategy outperform $1 / N$. 


\subsection{Performance in the 10Ind and 25SBM datasets}

I discuss the results for both datasets jointly (Tables 5,6) since similar conclusions can be drawn. Starting with panel A, the Sharpe ratios under no transaction costs confirm the findings of previous studies since all sample strategies apart from the tangency portfolio outperform $1 / N$. The largest Sharpe ratio is observed for the ICR strategy in the 10ind set (0.926) and for the 3F strategy in the $25 \mathrm{SBM}$ set (1.18). In both sets the Sharpe ratio of $1 / N$ is significantly lower $(0.542$ and 0.512 , respectively).

The results for the turnover again confirm the stability of $1 / N$ and the volatility timing strategy compared to other sample-based strategies. The turnover of these two portfolio ranges from 0.017 to 0.025 . The remaining strategies attain a turnover between 0.114 and 1.735 in the 10Ind set and between 0.320 and 38.19 in the 25SBM set. Clearly, the stability of the strategies deters with the number of assets since in this case estimation risk is higher. The most unstable strategies are the tangency portfolio, the norm-constrained portfolio and the portfolio based on the shrinkage of the inverse covariance under the returns criterion (ICR). Once I account for transaction costs, these strategies result to negative risk-adjusted returns. In this setting, $1 / N$ offers higher Sharpe ratio than most sample-based strategies. In the 10Ind dataset, only MIN, LW, VT and ICV perform better than naive diversification. In the 25SBM set, only LW and VT offer a higher Sharpe ratio. These findings again reveals the need for stable portfolios.

In panel B of Tables 5 and 6, I report the Sharpe ratios for the stable counterparts of all strategies. In the absence of transaction costs, the results are mixed with some strategies performing slightly better and other somewhat worse than their original analogues. However, under transaction costs of $100 \mathrm{bp}$, all stables strategies offer higher Sharpe ratios than $1 / N$. The most significant improvement is naturally observed for NC and ICR which are the most unstable strategies examined here. For example, in the 25SBM dataset the Sharpe ratio of ICR increases from -2 to 0.595 when the turnover is equal to that of $1 / N$ and to 0.596 when the stability parameter maximizes last period's portfolio return. As expected, the latter results to even more stable portfolios than $1 / N$ in terms of turnover. In general, the two selection criteria for the stability parameter lead to similar Sharpe ratios which are higher than $1 / N$.

The potential of the stability approach in improving portfolio performance is best illustrated by examining the results for the tangency portfolio in the 10Ind dataset. As discussed in section 2, this portfolio is very sensitive to estimation errors and very unstable by construction. This is clear by the results in Panel A; the Sharpe ratio is comparatively the lowest (0.289) and the turnover is more than 50 times that of $1 / N$. As a result, the tangency portfolio generates a negative Sharpe ratio net of transaction costs of -0.29. Imposing the stability penalty though dramatically improves

both the Sharpe ratio and the turnover. In fact, under the second criterion for selecting the stability parameter the tangency portfolio is most stable and efficient than $1 / N$ offering a Sharpe ratio of 0.5241 net of transaction costs and a turnover of 0.014 . 


\subsection{Performance in the 8Int and 50SP datasets}

The dataset of 8 international portfolios (Table 4) along with the 50 stocks dataset (Table 7) are the most challenging environment for testing the stability approach. According to the results in the first set (Table 4, Panel A), none of the sample strategies significantly outperforms $1 / N$ apart from the volatility timing strategy. Moreover, most of the strategies are very unstable compared to $1 / N$. The highest turnover again comes from the TP, 3F, NC and ICR portfolios $(8.355,20.41$, $0.97,1.973$, respectively). These strategies are disabled by transaction costs resulting in negative Sharpe ratios. Only the VT strategy outperforms $1 / N$ in the presence of transaction costs. In the 50SP set, the sample-based strategies perform even worse due to the large number of assets and the magnitude of estimation risk. All strategies apart from VT and NC perform worse than $1 / N$ in terms of both Sharpe ratio and turnover. Due to instability, most strategies result in negative Sharpe ratio under transaction costs of $100 \mathrm{bp}$. Therefore, it is interesting to see the extent to which the stability approach improves performance.

As Panel B of Tables 4 and 7 indicates, the stability penalty manages to drastically increase Sharpe ratio while reducing turnover. For the 8Int dataset, I find that the Sharpe ratio for all stable portfolios except from $\mathrm{TP}, 3 \mathrm{~F}$ and $\mathrm{LW}$ is higher than that of $1 / N$. This holds in both the presence and the absence of transaction costs. Optimizing the stability parameter further increases the Sharpe ratio. ICR and ICV and NC offer the highest Sharpe ratios of 0.419, 0.3592 compared to 0.327 of $1 / N$. Similar conclusions can be drawn by studying the respective results for the 50 stocks dataset (Table 7, Panel B). The stability approach improves all strategies in terms of Sharpe ratio and turnover, especially when the return-based stability criterion is employed. The highest Sharpe ratio net of transaction costs is obtained for the ICR strategy ( 0.75 compared to 0.6638 of $1 / N)$. Overall, the results confirm that the stability penalty is successful in producing portfolios that are both stable and efficient.

\section{Conclusions}

In this paper, I deal with the two main problems in the applicability of mean-variance portfolios: estimation risk and instability. I show that most existing sample-based strategies underperform $1 / N$ in the presence of transaction costs. This is because estimation errors increase instability as this is measured by the portfolio turnover. Motivated by this finding, I develop a new method to jointly treat estimation errors and instability. In particular, I propose to augment the investor's objective with a penalty that controls the deviation from the portfolio before rebalancing through a stability parameter.

The main advantage of my method compared to other approaches in the literature is that it leads to intuitive analytical representations of the portfolio weights. As such, it can effectively treat estimation risk since it can be readily applied to stabilise any efficient sample-based strategy. I further provide two interpretations to show that the stability penalty may improve performance even in the absence of transaction costs. To treat instability, I propose two selection criteria for the 
stability parameter. The first equalizes the turnover of the sample-based strategy to that of $1 / N$. The second maximizes last month's portfolio return on the basis of the reported momentum effect.

In my empirical analysis I apply the stability approach to several sample-based strategies from the literature. I find that the resulting stable portfolio strategies outperform $1 / N$ under most scenarios even in the presence of high transaction costs. At the same time, they offer a turnover which is equal or less than the turnover of $1 / N$. Among the two selection criteria for the stability parameter, the momentum-based parameter leads to higher Sharpe ratios and lower turnovers. Overall, my results confirm that the stability approach leads to stable and efficient portfolios. 


\section{References}

Best, M. J., and R. R. Grauer, 1991, "On the Sensitivity of Mean-Variance-Efficient Portfolios to Changes in Asset Means: Some Analytical and Computational Results," Review of Financial Studies, 4, 315-342.

Brandt, M. W., 2009. Portfolio choice problems. Handbook of financial econometrics: Tools and techniques, 269.

Campbell, J.Y. et al., 1997. The econometrics of financial markets, princeton University press Princeton, NJ.

Chopra, V. and W. Ziemba, 1993, "The Effect of Errors in Means, Variances, and Covariances on Optimal Portfolio Choice", Journal of Portfolio Management 19, 6-12.

DeMiguel, V., Garlappi, L., and Uppal, R., 2009a, "Optimal versus Naive Diversification: How Inefficient Is the 1/N Portfolio Strategy?" Review of Financial Studies, 22, 1915-1953.

DeMiguel, V., Garlappi, L., Nogales, F. J., and Uppal, R., 2009b. A generalized approach to portfolio optimization: Improving performance by constraining portfolio norms. Management Science, 55(5), 798-812.

DeMiguel, V., Nogales, F.J. and Uppal, R., 2012. Stock Return Serial Dependence and Out-ofSample Portfolio Performance. Working paper, London Business School.

Hayya, J., Armstrong, D. and Gressis, N., 1975, "A note on the ratio of two normally distributed variables," Management Science, 21, 1338-1341.

Hinkley, D.V., 1969. On the ratio of two correlated normal random variables. Biometrika, 56(3), 635-639.

Jagannathan, R., and T. Ma, 2003, "Risk Reduction in Large Portfolios: Why Imposing the Wrong Constraints Helps," Journal of Finance, 58, 1651-1684.

James,W. and C. Stein, 1961, "Estimation with quadratic loss", Proceedings of the 4th CityBerkeley Symposium on Mathematical Statistics and Probability 1, Berkeley, University of California Press, 361-379.

Kan, R and G. Zhou, 2007, "Optimal portfolio choice with parameter uncertainty," Journal of Financial and Quantitative Analysis, 42, 621-656.

Kan, R. and Smith, D.R., 2008. The Distribution of the Sample Minimum-Variance Frontier. Management Science, 54(7), 1364-1380.

Kourtis, A., Dotsis, G. and Markellos, R.N., 2012. Parameter uncertainty in portfolio selection: Shrinking the inverse covariance matrix. Journal of Banking and Finance 36, 2522-2531. 
Ledoit, O., and M. Wolf, 2003, "Improved Estimation of the Covariance Matrix of Stock Returns with an Application to Portfolio Selection," Journal of Empirical Finance, 10, 603-621.

Ledoit, O., and M. Wolf, 2004a, "Honey, I Shrunk the Sample Covariance Matrix: Problems in Mean-Variance Optimization," Journal of Portfolio Management, 30, 110-119.

Ledoit, O., and M. Wolf, 2004b, "A Well-Conditioned Estimator for Large-Dimensional Covariance Matrices," Journal of Multivariate Analysis, 88, 365-411

Ledoit, O. and Wolf, M., 2008. "Robust performance hypothesis testing with the Sharpe ratio", Journal of Empirical Finance, 15(5), 850-859.

Markowitz, H. M., 1952, "Portfolio Selection," Journal of Finance, 7, 77-91.

Marsaglia, G., 1965. Ratios of normal variables and ratios of sums of uniform variables. Journal of the American Statistical Association, 60(309), 193-204.

Merton, R. C., 1980, "On Estimating the expected return on the market: An exploratory Investigation," Journal of Financial Economics 8, 323-361.

Michaud, R. O., 1989, "The Markowitz Optimization Enigma: Is Optimized Optimal?" Financial Analysts Journal, 45, 31-42.

Tu, J. and Zhou, G., 2011. Markowitz meets Talmud: A combination of sophisticated and naive diversification strategies. Journal of Financial Economics, 99, 204-215.

Woodside-Oriakhi, M., Lucas, C. and Beasley, J.E., 2013. Portfolio rebalancing with an investment horizon and transaction costs. Omega 41, 406-420. 


\section{Table 1: List of portfolio strategies}

This table reports the portfolio strategies considered in the performance analysis.

\begin{tabular}{|c|c|}
\hline Abbreviation & Description \\
\hline $1 / N$ & Equal-weighted portfolio \\
\hline $\mathbf{T P}$ & Sample tangency portfolio \\
\hline MIN & Sample global minimum variance portfolio \\
\hline MV & Sample mean-variance portfolio with a required return equal to the return of $1 / N$ \\
\hline MVC & $\begin{array}{l}\text { Short-sale constrained mean variance portfolio with a required return equal to the return of } \\
1 / N\end{array}$ \\
\hline LW & $\begin{array}{l}\text { Minimum variance portfolio that results from shrinking the covariance matrix to the identity } \\
\text { (Ledoit and Wolf } 2004 \mathrm{~b} \text { ) }\end{array}$ \\
\hline NC & $\begin{array}{l}\text { 2-norm constrained minimum variance portfolio that exploits the positive autocorrelation of } \\
\text { portfolio returns (DeMiguel et al. 2009b) }\end{array}$ \\
\hline $3 \mathbf{F}$ & The linear combination of MEAN, GMV and $1 / N$ portfolios proposed in Tu and Zhou (2011) \\
\hline VT & $\begin{array}{l}\text { Minimum variance portfolio that results from using a diagonal covariance matrix (Kirby and } \\
\text { Ostdiek, 2012) }\end{array}$ \\
\hline ICV & $\begin{array}{l}\text { Minimum variance portfolio that results from shrinking the ML estimator of the inverse co- } \\
\text { variance matrix to a linear combination the identity and the inverse covariance matrix from } \\
\text { a 1-factor model. The shrinkage parameters minimize out-of-sample variance (Kourtis et al. } \\
\text { 2012) }\end{array}$ \\
\hline ICR & $\begin{array}{l}\text { Minimum variance portfolio that results from shrinking the ML estimator of the inverse co- } \\
\text { variance matrix to a linear combination of the identity and the inverse covariance matrix from } \\
\text { a 1-factor model. The shrinkage parameters maximize last period's return (Kourtis et al. } \\
2012 \text { ) }\end{array}$ \\
\hline
\end{tabular}




\section{Table 2: List of datasets}

This table lists the datasets of monthly excess returns that I use for the performance analysis along with the time period they span. All data are obtained from Kenneth French's website (http://mba.tuck.dartmouth.edu/pages/faculty/ken.french/data_library.html) apart from the stocks dataset which is obtained by Google. I use the 30-day T-bill rate, also obtained from Kenneth French's website, for the derivation of excess returns

\begin{tabular}{|c|c|c|}
\hline Abbreviation & Time Period & Description \\
\hline $3 \mathrm{FF}$ & 07/1969-09/2012 & 3 Fama-French factors \\
\hline $8 \operatorname{Int}$ & 01/1981-12/2011 & $\begin{array}{l}8 \text { international market portfolios (Australia, } \\
\text { Canada, France, Germany, Japan, Italy, UK } \\
\text { and USA) }\end{array}$ \\
\hline 10Ind & 07/1969-09/2012 & 10 industry portfolios \\
\hline $25 \mathrm{FF}$ & 07/1969-09/2012 & 25 size and book-to-market portfolios \\
\hline 50S\&P & 01/1981-10/2012 & $50 \mathrm{~S} \& \mathrm{P} 500$ stocks \\
\hline
\end{tabular}




\section{Table 3: Results for the 3 Fama French Dataset}

This table summarizes the out-of-sample performance of the strategies described in Table 1 in the 3 Fama French dataset. Panel A reports the average return, variance and Sharpe ratio in annual terms and the average turnover. It also reports the Sharpe ratio in the presence of proportional transaction costs of 100 basis points. All these measures are derived using a rolling window approach for a sample size of 120 months. One, two or three stars next to the Sharpe ratio of the sample-based strategies indicate that the difference from the Sharpe ratio of $1 / N$ is statistically significant at the 10\%, 5\% and 1\% level, respectively. This hypothesis test is performed using a studentized circular block bootstrap method with 1000 trials and an expected block size of 5. Panel B reports the same metrics as in Panel A for the stable counterparts of the strategies of Table 1. Stability is enforced by setting the turnover of the sample-based strategies equal to the turnover of $1 / N$ as described in the text. The table also reports in parenthesis the Sharpe ratio and turnover when stability is enforced so that it maximizes last month's portfolio return (see text for more details).

\begin{tabular}{|c|c|c|c|c|c|c|c|}
\hline \multirow[b]{2}{*}{ Strategy } & \multicolumn{5}{|c|}{ No transaction costs } & \multirow{2}{*}{\multicolumn{2}{|c|}{$\begin{array}{c}\text { Transaction costs } \\
\hat{\theta}^{p}\end{array}$}} \\
\hline & \multirow{2}{*}{$\begin{array}{l}\hat{\mu}^{p} \\
0.0547\end{array}$} & \multirow{2}{*}{$\begin{array}{l}\left(\hat{\sigma}^{p}\right)^{2} \\
0.0042\end{array}$} & \multirow{2}{*}{$\begin{array}{l}\hat{\theta}^{p} \\
0.8405\end{array}$} & \multicolumn{2}{|c|}{$\hat{\tau}^{p}$} & & \\
\hline $1 / N$ & & & & 0.0229 & & \multirow{2}{*}{0.7983} & \\
\hline \multicolumn{7}{|c|}{ Panel A: Original strategies } & \\
\hline $\mathrm{TP}$ & 0.0651 & 0.0051 & 0.9143 & 0.0509 & & 0.8289 & \\
\hline MIN & 0.0415 & 0.0032 & 0.7336 & 0.0243 & & 0.6833 & \\
\hline MV & 0.0518 & 0.0033 & $0.8966^{*}$ & 0.0358 & & 0.8232 & \\
\hline MVC & 0.0518 & 0.0033 & $0.8966^{*}$ & 0.0358 & & 0.8232 & \\
\hline LW & 0.0424 & 0.0032 & 0.7473 & 0.0237 & & 0.6983 & \\
\hline $\mathrm{NC}$ & 0.0562 & 0.0034 & $0.9613^{* * *}$ & 0.1609 & & $0.6263^{*}$ & \\
\hline $3 \mathrm{~F}$ & 0.0608 & 0.0041 & $0.9455^{* *}$ & 0.0360 & & $0.8795^{*}$ & \\
\hline $\mathrm{VT}$ & 0.0348 & 0.0034 & $0.5956^{* *}$ & 0.0231 & & $0.5492 * *$ & \\
\hline $\mathrm{ICV}$ & 0.0404 & 0.0032 & $0.7150^{*}$ & 0.0252 & & $0.6627^{*}$ & \\
\hline ICR & 0.0457 & 0.0047 & $0.6662^{*}$ & 0.4109 & & $-0.0520 * * *$ & \\
\hline \multicolumn{8}{|c|}{ Panel B: Stable strategies } \\
\hline $\mathrm{TP}$ & 0.0678 & 0.0053 & 0.9283 & 0.0229 & $(0.0091)$ & 0.8910 & $(0.9666)$ \\
\hline MIN & 0.0419 & 0.0032 & 0.7380 & 0.0229 & $(0.0059)$ & 0.6899 & $(0.8278)$ \\
\hline MV & 0.0538 & 0.0034 & $0.9280^{*}$ & 0.0229 & $(0.0059)$ & $0.8809^{*}$ & $\left(0.9492^{* *}\right)$ \\
\hline MVC & 0.0527 & 0.0033 & $0.9148^{*}$ & 0.0229 & $(0.0073)$ & $0.8674^{*}$ & $(0.9399 * * *)$ \\
\hline LW & 0.0432 & 0.0033 & 0.7543 & 0.0229 & $(0.0059)$ & 0.7066 & $\left(0.8404^{*}\right)$ \\
\hline $\mathrm{NC}$ & 0.0547 & 0.0038 & $0.8841^{*}$ & 0.0229 & $(0.0142)$ & $0.8401^{*}$ & $\left(0.8737^{* *}\right)$ \\
\hline $3 \mathrm{~F}$ & 0.0650 & 0.0043 & $0.9850 * * *$ & 0.0229 & $(0.0079)$ & $0.9438 * * *$ & $\left(0.9963^{* * *}\right)$ \\
\hline $\mathrm{VT}$ & 0.0352 & 0.0035 & $0.5985^{*}$ & 0.0229 & $(0.0070)$ & $0.5520 *$ & $(0.7050)$ \\
\hline ICV & 0.0405 & 0.0032 & 0.7146 & 0.0229 & $(0.0061)$ & 0.6664 & $(0.8099)$ \\
\hline ICR & 0.0572 & 0.0040 & $0.9088^{* *}$ & 0.0229 & $(0.0155)$ & $0.8660^{* *}$ & $\left(0.8812^{* *}\right)$ \\
\hline
\end{tabular}


Table 4: Results for the 8 International Portfolios Dataset

This table summarizes the out-of-sample performance of the strategies described in Table 1 in the 8 international portfolios dataset. Panel A reports the average return, variance and Sharpe ratio in annual terms and the average turnover. It also reports the Sharpe ratio in the presence of proportional transaction costs of 100 basis points. All these measures are derived using a rolling window approach for a sample size of 120 months. One, two or three stars next to the Sharpe ratio of the sample-based strategies indicate that the difference from the Sharpe ratio of $1 / N$ is statistically significant at the $10 \%, 5 \%$ and $1 \%$ level, respectively. This hypothesis test is performed using a studentized circular block bootstrap method with 1000 trials and an expected block size of 5 . Panel B reports the same metrics as in Panel A for the stable counterparts of the strategies of Table 1. Stability is enforced by setting the turnover of the sample-based strategies equal to the turnover of $1 / N$ as described in the text. The table also reports in parenthesis the Sharpe ratio and turnover when stability is enforced so that it maximizes last month's portfolio return (see text for more details).

\begin{tabular}{|c|c|c|c|c|c|c|c|}
\hline \multirow{3}{*}{$\frac{\text { Strategy }}{1 / N}$} & \multicolumn{5}{|c|}{ No transaction costs } & \multirow{2}{*}{\multicolumn{2}{|c|}{$\begin{array}{c}\text { Transaction costs } \\
\hat{\theta}^{p}\end{array}$}} \\
\hline & $\hat{\mu}^{p}$ & $\left(\hat{\sigma}^{p}\right)^{2}$ & $\hat{\theta}^{p}$ & \multicolumn{2}{|c|}{$\overline{\hat{\tau}^{p}}$} & & \\
\hline & 0.0577 & 0.0282 & 0.3438 & 0.0241 & & 0.3265 & \\
\hline \multicolumn{8}{|c|}{ Panel A: Original strategies } \\
\hline $\mathrm{TP}$ & 0.0956 & 1.8203 & $0.0708^{*}$ & 8.3554 & & $-0.2808^{* * *}$ & \\
\hline MIN & 0.0432 & 0.0203 & 0.3031 & 0.1190 & & $0.2026^{* *}$ & \\
\hline MV & 0.0553 & 0.0248 & 0.3512 & 0.1650 & & $0.2250^{*}$ & \\
\hline MVC & 0.0569 & 0.0256 & 0.3556 & 0.0939 & & 0.2850 & \\
\hline LW & 0.0453 & 0.0204 & 0.3171 & 0.0833 & & $0.2469 *$ & \\
\hline $\mathrm{NC}$ & 0.0438 & 0.0231 & $0.2881^{*}$ & 0.9667 & & $-0.4722^{* * *}$ & \\
\hline $3 \mathrm{~F}$ & 0.5332 & 13.2279 & $0.1466^{* *}$ & 20.4056 & & $-0.2174^{* * *}$ & \\
\hline $\mathrm{VT}$ & 0.0596 & 0.0265 & 0.3660 & 0.0242 & & 0.3482 & \\
\hline ICV & 0.0500 & 0.0205 & 0.3486 & 0.0992 & & $0.2652^{*}$ & \\
\hline ICR & 0.0275 & 0.0268 & $0.1677^{* *}$ & 1.9726 & & $-1.2080^{* *}$ & \\
\hline \multicolumn{8}{|c|}{ Panel B: Stable strategies } \\
\hline $\mathrm{TP}$ & 0.2236 & 0.5427 & $0.3035^{*}$ & 0.0241 & $(0.0157)$ & $0.2995^{*}$ & $\left(0.2043^{*}\right)$ \\
\hline MIN & 0.0528 & 0.0225 & 0.3525 & 0.0241 & $(0.0094)$ & 0.3332 & $(0.3754)$ \\
\hline MV & 0.0570 & 0.0237 & $0.3704^{*}$ & 0.0241 & $(0.0112)$ & 0.3516 & $(0.3995)$ \\
\hline MVC & 0.0595 & 0.0264 & 0.3660 & 0.0241 & $(0.0241)$ & 0.3482 & $(0.3529)$ \\
\hline LW & 0.0509 & 0.0221 & 0.3427 & 0.0241 & $(0.0241)$ & 0.3232 & $(0.3232)$ \\
\hline $\mathrm{NC}$ & 0.0599 & 0.0265 & 0.3681 & 0.0241 & $(0.0203)$ & 0.3503 & $(0.3551)$ \\
\hline $3 \mathrm{~F}$ & 0.0544 & 0.0283 & 0.3237 & 0.0241 & $(0.0102)$ & 0.3065 & $(0.3204)$ \\
\hline $\mathrm{VT}$ & 0.0598 & 0.0266 & 0.3670 & 0.0241 & $(0.0060)$ & 0.3493 & $(0.3627)$ \\
\hline $\mathrm{ICV}$ & 0.0570 & 0.0227 & $0.3784^{*}$ & 0.0241 & $(0.0241)$ & $0.3592^{*}$ & $\left(0.3592^{*}\right)$ \\
\hline ICR & 0.0709 & 0.0277 & $0.4264^{* *}$ & 0.0241 & $(0.0209)$ & $0.4090 * *$ & $\left(0.4185^{* *}\right)$ \\
\hline
\end{tabular}




\section{Table 5: Results for the 10 Industry Portfolios Dataset}

This table summarizes the out-of-sample performance of the strategies described in Table 1 in the 10 industry portfolios dataset. Panel A reports the average return, variance and Sharpe ratio in annual terms and the average turnover. It also reports the Sharpe ratio in the presence of proportional transaction costs of 100 basis points. All these measures are derived using a rolling window approach for a sample size of 120 months. One, two or three stars next to the Sharpe ratio of the sample-based strategies indicate that the difference from the Sharpe ratio of $1 / N$ is statistically significant at the $10 \%, 5 \%$ and $1 \%$ level, respectively. This hypothesis test is performed using a studentized circular block bootstrap method with 1000 trials and an expected block size of 5 . Panel B reports the same metrics as in Panel A for the stable counterparts of the strategies of Table 1. Stability is enforced by setting the turnover of the sample-based strategies equal to the turnover of $1 / N$ as described in the text. The table also reports in parenthesis the Sharpe ratio and turnover when stability is enforced so that it maximizes last month's portfolio return (see text for more details).

\begin{tabular}{|c|c|c|c|c|c|c|c|}
\hline \multirow[b]{2}{*}{ Strategy } & \multicolumn{5}{|c|}{ No transaction costs } & \multicolumn{2}{|c|}{ Transaction costs } \\
\hline & \multirow{2}{*}{$\begin{array}{l}\hat{\mu}^{p} \\
0.0804\end{array}$} & \multirow{2}{*}{$\begin{array}{l}\left(\hat{\sigma}^{p}\right)^{2} \\
0.0220\end{array}$} & \multirow{2}{*}{$\begin{array}{l}\hat{\theta}^{p} \\
0.5418\end{array}$} & \multicolumn{2}{|c|}{$\hat{\tau}^{p}$} & \multicolumn{2}{|c|}{$\hat{\theta}^{p}$} \\
\hline $1 / N$ & & & & \multicolumn{2}{|l|}{0.0240} & \multicolumn{2}{|l|}{0.5223} \\
\hline \multicolumn{8}{|c|}{ Panel A: Original strategies } \\
\hline $\mathrm{TP}$ & 0.0774 & 0.0718 & $0.2889^{*}$ & 1.3037 & & -0.2954 & \\
\hline MIN & 0.0865 & 0.0148 & $0.7107^{*}$ & 0.1570 & & 0.5557 & \\
\hline MV & 0.0848 & 0.0159 & $0.6734^{*}$ & 0.2160 & & $0.4665^{*}$ & \\
\hline MVC & 0.0766 & 0.0156 & 0.6130 & 0.1143 & & 0.5032 & \\
\hline LW & 0.0849 & 0.0143 & $0.7114^{*}$ & 0.1066 & & 0.6044 & \\
\hline $\mathrm{NC}$ & 0.1053 & 0.0164 & $0.8221^{* *}$ & 0.9483 & & $-0.0755^{* * *}$ & \\
\hline $3 \mathrm{~F}$ & 0.0817 & 0.0170 & 0.6257 & 0.1797 & & $0.4613^{*}$ & \\
\hline $\mathrm{VT}$ & 0.0818 & 0.0189 & 0.5945 & 0.0249 & & 0.5727 & \\
\hline $\mathrm{ICV}$ & 0.0868 & 0.0146 & $0.7191^{*}$ & 0.1142 & & $0.6058^{*}$ & \\
\hline ICR & 0.1499 & 0.0262 & $0.9256^{* * *}$ & 1.7349 & & $-0.3753^{* * *}$ & \\
\hline \multicolumn{8}{|c|}{ Panel B: Stable strategies } \\
\hline $\mathrm{TP}$ & 0.1476 & 0.1201 & $0.4260 *$ & 0.0240 & $(0.0141)$ & 0.4178 & $(0.5241)$ \\
\hline MIN & 0.0897 & 0.0153 & $0.7251^{* *}$ & 0.0240 & $(0.0112)$ & 0.7017 & $(0.6853)$ \\
\hline MV & 0.0896 & 0.0173 & $0.6806^{* *}$ & 0.0240 & $(0.0106)$ & $0.6586^{* *}$ & $\left(0.6371^{* *}\right)$ \\
\hline MVC & 0.0781 & 0.0163 & $0.6115^{*}$ & 0.0240 & $(0.0124)$ & $0.5889^{*}$ & $\left(0.5824^{*}\right)$ \\
\hline LW & 0.0844 & 0.0146 & $0.6997^{* *}$ & 0.0240 & $(0.0116)$ & $0.6757^{* *}$ & $\left(0.6774^{* *}\right)$ \\
\hline $\mathrm{NC}$ & 0.0820 & 0.0178 & $0.6150^{*}$ & 0.0240 & $(0.0205)$ & $0.5933^{*}$ & $\left(0.5996^{*}\right)$ \\
\hline $3 \mathrm{~F}$ & 0.0798 & 0.0172 & 0.6083 & 0.0240 & $(0.0127)$ & 0.5863 & $(0.5826)$ \\
\hline $\mathrm{VT}$ & 0.0819 & 0.0190 & 0.5946 & 0.0240 & $(0.0058)$ & 0.5736 & $(0.5855)$ \\
\hline ICV & 0.0857 & 0.0148 & $0.7044^{* *}$ & 0.0240 & $(0.0115)$ & $0.6806^{* *}$ & $\left(0.6854^{* *}\right)$ \\
\hline ICR & 0.0840 & 0.0166 & $0.6512^{* *}$ & 0.0240 & $(0.0217)$ & $0.6287^{* *}$ & $\left(0.6286^{* *}\right)$ \\
\hline
\end{tabular}


Table 6: Results for the 25 Size and Book-to-Market Portfolios Dataset

This table summarizes the out-of-sample performance of the strategies described in Table 1 in the 25 size and book-to-market dataset. Panel A reports the average return, variance and Sharpe ratio in annual terms and the average turnover. It also reports the Sharpe ratio in the presence of proportional transaction costs of 100 basis points. All these measures are derived using a rolling window approach for a sample size of 120 months. One, two or three stars next to the Sharpe ratio of the sample-based strategies indicate that the difference from the Sharpe ratio of $1 / N$ is statistically significant at the $10 \%, 5 \%$ and $1 \%$ level, respectively. This hypothesis test is performed using a studentized circular block bootstrap method with 1000 trials and an expected block size of 5 . Panel B reports the same metrics as in Panel A for the stable counterparts of the strategies of Table 1. Stability is enforced by setting the turnover of the sample-based strategies equal to the turnover of $1 / N$ as described in the text. The table also reports in parenthesis the Sharpe ratio and turnover when stability is enforced so that it maximizes last month's portfolio return (see text for more details).

\begin{tabular}{|c|c|c|c|c|c|c|c|}
\hline \multirow[b]{2}{*}{ Strategy } & \multicolumn{5}{|c|}{ No transaction costs } & \multicolumn{2}{|c|}{ Transaction costs } \\
\hline & \multirow{2}{*}{$\begin{array}{l}\hat{\mu}^{p} \\
0.0893\end{array}$} & \multirow{2}{*}{$\begin{array}{l}\left(\hat{\sigma}^{p}\right)^{2} \\
0.0304\end{array}$} & \multirow{2}{*}{$\begin{array}{l}\hat{\theta}^{p} \\
0.5123\end{array}$} & \multicolumn{2}{|c|}{$\hat{\tau}^{p}$} & \multicolumn{2}{|c|}{$\hat{\theta}^{p}$} \\
\hline $1 / N$ & & & & 0.0173 & & 0.5004 & \\
\hline \multicolumn{8}{|c|}{ Panel A: Original strategies } \\
\hline $\mathrm{TP}$ & 0.4869 & 0.5918 & $0.6329^{*}$ & 38.1853 & & $-0.3266^{* * *}$ & \\
\hline MIN & 0.1448 & 0.0177 & $1.0882^{* * *}$ & 0.8140 & & $0.3462^{* *}$ & \\
\hline MV & 0.1428 & 0.0177 & $1.0722^{* * *}$ & 0.8623 & & $0.2870^{* *}$ & \\
\hline MVC & 0.1282 & 0.0344 & $0.6919 *$ & 0.5008 & & $0.3595^{*}$ & \\
\hline LW & 0.1212 & 0.0159 & $0.9617^{* * *}$ & 0.3197 & & 0.6552 & \\
\hline $\mathrm{NC}$ & 0.1416 & 0.0197 & $1.0098^{* * *}$ & 3.7935 & & $-1.8537^{* * *}$ & \\
\hline $3 \mathrm{~F}$ & 0.2010 & 0.0289 & $1.1826 * * *$ & 1.3717 & & $0.1968^{*}$ & \\
\hline $\mathrm{VT}$ & 0.0915 & 0.0279 & 0.5481 & 0.0182 & & 0.5351 & \\
\hline ICV & 0.1267 & 0.0169 & $0.9754^{* * *}$ & 0.5659 & & 0.4488 & \\
\hline ICR & 0.1917 & 0.0376 & $0.9881^{* * *}$ & 5.3635 & & $-2.0003^{* * *}$ & \\
\hline \multicolumn{8}{|c|}{ Panel B: Stable strategies } \\
\hline $\mathrm{TP}$ & 0.2561 & 0.3141 & 0.4570 & 0.0173 & $(0.0117)$ & 0.4533 & $(0.4466)$ \\
\hline MIN & 0.1158 & 0.0208 & $0.8025^{* * *}$ & 0.0173 & $(0.0094)$ & $0.7881^{* * *}$ & $\left(0.7533^{* *}\right)$ \\
\hline MV & 0.1175 & 0.0219 & $0.7950 * * *$ & 0.0173 & $(0.0082)$ & $0.7809^{* * *}$ & $\left(0.7474^{* *}\right)$ \\
\hline MVC & 0.0845 & 0.0255 & 0.5291 & 0.0173 & $(0.0102)$ & 0.5160 & $(0.5299)$ \\
\hline LW & 0.1094 & 0.0201 & $0.7707^{* *}$ & 0.0173 & $(0.0101)$ & $0.7560 * * *$ & $\left(0.7189^{* *}\right)$ \\
\hline $\mathrm{NC}$ & 0.0978 & 0.0271 & $0.5941^{*}$ & 0.0173 & $(0.0157)$ & 0.5814 & $(0.5872)$ \\
\hline $3 \mathrm{~F}$ & 0.1121 & 0.0288 & $0.6607^{* *}$ & 0.0173 & $(0.0115)$ & $0.6484^{* *}$ & $\left(0.6205^{* *}\right)$ \\
\hline $\mathrm{VT}$ & 0.0914 & 0.0279 & $0.5473^{*}$ & 0.0173 & $(0.0043)$ & $0.5348^{*}$ & $\left(0.5471^{*}\right)$ \\
\hline ICV & 0.1117 & 0.0202 & $0.7865^{* *}$ & 0.0173 & $(0.0093)$ & $0.7718^{* *}$ & $\left(0.7407^{* *}\right)$ \\
\hline ICR & 0.0984 & 0.0262 & $0.6082^{*}$ & 0.0173 & $(0.0160)$ & $0.5953^{* *}$ & $(0.5959 * *)$ \\
\hline
\end{tabular}




\section{Table 7: Results for the 50 S\&P500 Stocks Dataset}

This table summarizes the out-of-sample performance of the strategies described in Table 1 in the 50 S\&P500 stocks dataset. Panel A reports the average return, variance and Sharpe ratio in annual terms and the average turnover. It also reports the Sharpe ratio in the presence of proportional transaction costs of 100 basis points. All these measures are derived using a rolling window approach for a sample size of 120 months. One, two or three stars next to the Sharpe ratio of the sample-based strategies indicate that the difference from the Sharpe ratio of $1 / N$ is statistically significant at the 10\%, 5\% and 1\% level, respectively. This hypothesis test is performed using a studentized circular block bootstrap method with 1000 trials and an expected block size of 5. Panel B reports the same metrics as in Panel A for the stable counterparts of the strategies of Table 1. Stability is enforced by setting the turnover of the sample-based strategies equal to the turnover of $1 / N$ as described in the text. The table also reports in parenthesis the Sharpe ratio and turnover when stability is enforced so that it maximizes last month's portfolio return (see text for more details).

\begin{tabular}{|c|c|c|c|c|c|c|c|}
\hline \multirow[b]{2}{*}{ Strategy } & \multicolumn{5}{|c|}{ No transaction costs } & \multicolumn{2}{|c|}{ Transaction costs } \\
\hline & \multirow{2}{*}{$\begin{array}{l}\hat{\mu}^{p} \\
0.1074\end{array}$} & \multirow{2}{*}{$\begin{array}{l}\left(\hat{\sigma}^{p}\right)^{2} \\
0.0233\end{array}$} & \multirow{2}{*}{$\begin{array}{l}\hat{\theta}^{p} \\
0.7031\end{array}$} & \multicolumn{2}{|c|}{$\hat{\tau}^{p}$} & \multicolumn{2}{|c|}{$\hat{\theta}^{p}$} \\
\hline $1 / N$ & & & & 0.0501 & & 0.6638 & \\
\hline \multicolumn{8}{|c|}{ Panel A: Original strategies } \\
\hline $\mathrm{TP}$ & -0.0253 & 0.1658 & $-0.0621 * * *$ & 2.7254 & & $-0.8265^{* * *}$ & \\
\hline MIN & 0.0352 & 0.0223 & $0.2353^{* *}$ & 0.5227 & & $-0.1862^{* * *}$ & \\
\hline MV & 0.0346 & 0.0222 & $0.2323^{* *}$ & 0.5326 & & $-0.1991^{* * *}$ & \\
\hline MVC & 0.0726 & 0.0135 & $0.6245^{*}$ & 0.1284 & & $0.4919 * *$ & \\
\hline LW & 0.0661 & 0.0135 & $0.5685^{*}$ & 0.2133 & & $0.3475^{* *}$ & \\
\hline $\mathrm{NC}$ & 0.0992 & 0.0174 & 0.7518 & 1.6295 & & $-0.7181^{* * *}$ & \\
\hline $3 \mathrm{~F}$ & 0.0641 & 0.0220 & $0.4319^{* *}$ & 0.5765 & & $-0.0352^{* * *}$ & \\
\hline $\mathrm{VT}$ & 0.0970 & 0.0178 & $0.7270^{*}$ & 0.0479 & & $0.6841^{*}$ & \\
\hline ICV & 0.0698 & 0.0134 & $0.6039^{*}$ & 0.2033 & & $0.3921^{* *}$ & \\
\hline ICR & 0.1008 & 0.0311 & $0.5718^{* *}$ & 2.3141 & & $-0.9999 * * *$ & \\
\hline \multicolumn{8}{|c|}{ Panel B: Stable strategies } \\
\hline $\mathrm{TP}$ & 0.5950 & 12.7863 & $0.1664^{* *}$ & 0.0501 & $(0.0293)$ & $0.1647^{* *}$ & $\left(0.0777^{* *}\right)$ \\
\hline MIN & 0.0764 & 0.0328 & $0.4215^{* *}$ & 0.0501 & $(0.0237)$ & $0.3883^{* *}$ & $\left(0.4022^{* *}\right)$ \\
\hline MV & 0.0842 & 0.0479 & $0.3849^{* *}$ & 0.0501 & $(0.0236)$ & $0.3574^{* *}$ & $\left(0.3769^{* *}\right)$ \\
\hline MVC & 0.0748 & 0.0139 & 0.6342 & 0.0501 & $(0.0216)$ & 0.5831 & $(0.6018)$ \\
\hline LW & 0.0737 & 0.0154 & $0.5943^{*}$ & 0.0501 & $(0.0226)$ & $0.5457^{*}$ & $\left(0.5164^{*}\right)$ \\
\hline $\mathrm{NC}$ & 0.0934 & 0.0162 & $0.7342^{*}$ & 0.0501 & $(0.0385)$ & $0.6868^{*}$ & $\left(0.7040^{*}\right)$ \\
\hline $3 \mathrm{~F}$ & 0.0923 & 0.0184 & 0.6802 & 0.0501 & $(0.0265)$ & 0.6358 & $(0.6749)$ \\
\hline $\mathrm{VT}$ & 0.0972 & 0.0179 & $0.7260^{*}$ & 0.0501 & $(0.0081)$ & $0.6810^{*}$ & $\left(0.7297^{*}\right)$ \\
\hline ICV & 0.0751 & 0.0138 & $0.6381^{*}$ & 0.0501 & $(0.0196)$ & $0.5868^{*}$ & $(0.6145)$ \\
\hline ICR & 0.0932 & 0.0144 & $0.7764^{* *}$ & 0.0501 & $(0.0414)$ & $0.7260 * *$ & $\left(0.7505^{* *}\right)$ \\
\hline
\end{tabular}




\section{Table 8: Performance of optimal portfolios under transaction costs}

This table reports the Sharpe ratio in annual terms (Panel A) and the average turnover (Panel B) for the equal-weighted portfolio and two mean-variance portfolios that are optimal under proportional transaction costs of 100 basis points. These measures are derived using a rolling window approach for a sample size of 120 months. One, two or three stars next to the Sharpe ratio of the sample-based strategies indicate that the difference from the Sharpe ratio of $1 / N$ is statistically significant at the $10 \%, 5 \%$ and $1 \%$ level, respectively. This hypothesis test is performed using a studentized circular block bootstrap method with 1000 trials and an expected block size of 5. MV denotes the mean-variance portfolio with a required level of return net of transaction costs equal to the return of the equal weighted portfolio. MVC is the short-sale constrained counterpart of MV.

\begin{tabular}{clllll}
\hline Strategy & $3 \mathrm{FF}$ & 8Int & \multicolumn{1}{c}{ 10Ind } & 25SBM & $50 \mathrm{SP}$ \\
& \multicolumn{5}{c}{ Panel A: Sharpe Ratio } \\
\hline $1 / N$ & 0.7983 & 0.3265 & 0.5223 & 0.5004 & 0.6638 \\
MV & $0.8658^{*}$ & 0.3020 & $0.7065^{* *}$ & $0.9132^{* * *}$ & $0.2052^{*}$ \\
MVC & $0.9096^{* *}$ & 0.3241 & $0.6120^{*}$ & $0.1405^{* *}$ & 0.6016 \\
& & \multicolumn{5}{c}{ Panel B: Turnover } \\
\hline MV & 0.0229 & 0.0241 & 0.0240 & 0.0173 & 0.0501 \\
MVC & 0.0100 & 0.0236 & 0.0329 & 0.0382 & 0.1423 \\
& 0.0083 & 0.0156 & 0.0165 & 0.6350 & 0.0651 \\
\hline
\end{tabular}


Table 9: Distributional properties of the turnover of $1 / N$

This table contains a set of useful statistics (Panel A) that enable the approximation of the distribution of the turnover as this is derived in subsection 4.3. Panel B contains the average turnover of the $1 / N$ portfolio for each dataset of Table 2 and its estimator computed using the suggestions in subsection 4.3. It also reports the standard error of the estimator.

\begin{tabular}{rrrrrr}
\hline Statisic & 3FF & 8Int & 10Ind & $25 \mathrm{FF}$ & 50SP \\
& Panel A: Useful statistics & & \\
\hline$c v(X)$ & 0.018581 & 0.047835 & 0.042277 & 0.049593 & 0.0435 \\
$c v\left(Y_{i}\right)$ & 24.66742 & 63.05045 & 50.02501 & 49.84192 & 165.0798 \\
$\min \left(c v\left(Y_{i}\right)\right)$ & 5.335742 & 8.93878 & 12.90212 & 6.393113 & 6.775161 \\
$\bar{\rho}\left(X, Y_{i}\right)$ & 0.3324 & 0.214677 & 0.264381 & 0.236444 & 0.227137 \\
$\max \left(\rho\left(X, Y_{i}\right)\right)$ & 0.49174 & 0.377236 & 0.514432 & 0.489793 & 0.548175 \\
Panel B: Turnover estimators for $1 / N$ & & \\
\hline$\tau^{1 / N}$ & 0.0229 & 0.0241 & 0.0240 & 0.0173 & 0.0501 \\
$\tilde{\tau}^{1 / N}$ & 0.0248 & 0.0290 & 0.0243 & 0.0190 & 0.0537 \\
S.E & 0.0019 & 0.0049 & 0.0003 & 0.0016 & 0.0036 \\
\hline
\end{tabular}


Figure 1: True vs approximate cdf of the turnover for the $1 / N$ portfolio

This figure shows the real cumulative distribution function (cdf) of the turnover for the first asset of the $1 / N$ portfolio and the approximate cdf derived in subsection 4.3 in the paper. Panels A, B, C and D correspond to the 8Int, 10Ind, 25SBM and 50SP datasets (see Table 2 for a description). Both cdfs are derived under the assumption of the asset returns follow a multivariate normal distribution with mean and covariance equal to their sample counterparts. For the construction of the cdfs 10,000 random normal observations were extracted from the returns distribution.

Panel A

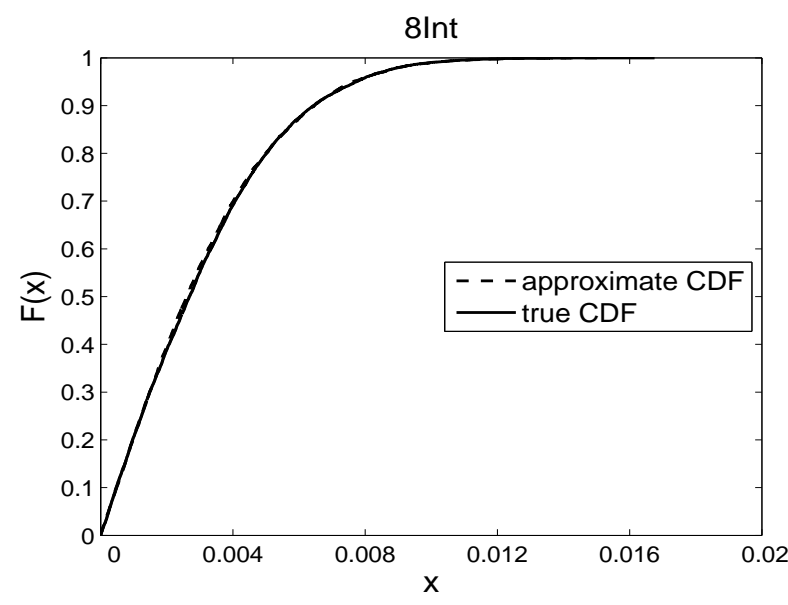

Panel C

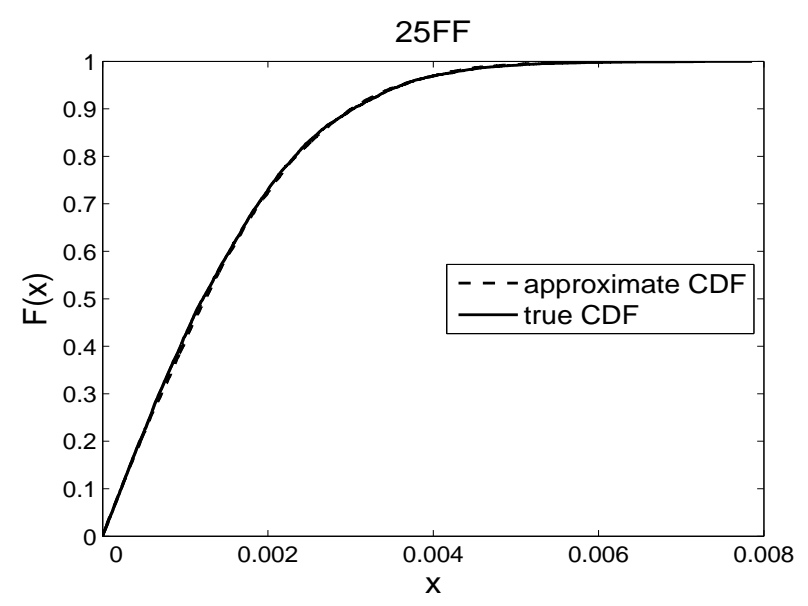

Panel B

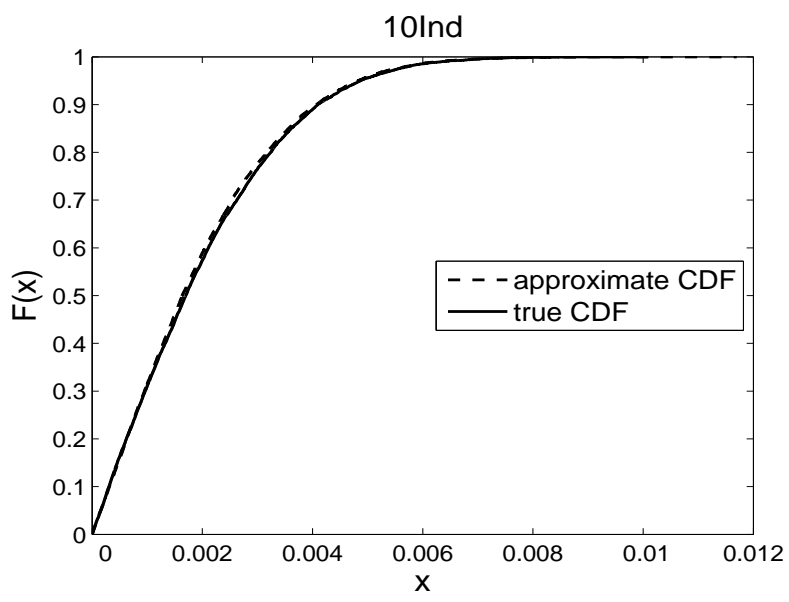

Panel D

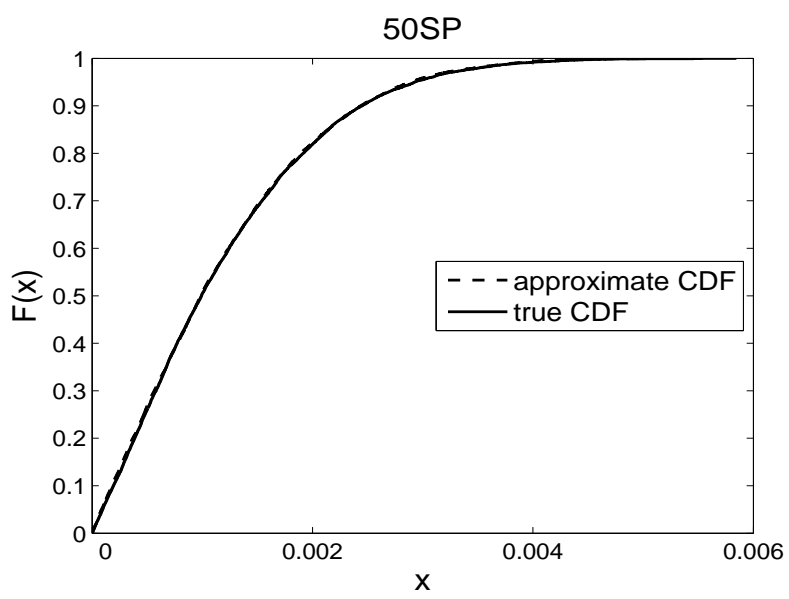

